\title{
Isolation, Molecular Characterization and Antibiotic Susceptibility Pattern of Vibrio parahaemolyticus from Aquatic Products in the Southern Fujian Coast, China
}

\author{
Yuanqing $\mathrm{Hu}^{1,2 *}$, Fengxia Li', Yixian Zheng ${ }^{1}$, Xinan Jiao ${ }^{2}$, and Liqing $\mathrm{Guo}^{3}$ \\ ${ }^{1}$ School of Biological Science and Biotechnology, Minnan Normal University, Zhangzhou 363000, P.R. China \\ ${ }^{2}$ Jiangsu Key Laboratory of Zoonosis, Yangzhou University, Yangzhou 225000, P.R. China \\ ${ }^{3}$ Zhangzhou Center for Disease Control and Prevention, Zhangzhou 363000, P.R. China
}

\begin{abstract}
Vibrio parahaemolyticus is a major gastroenteritis-causing pathogen in many Asian countries. Antimicrobial resistance in $V$. parahaemolyticus has been recognized as a critical threat to food safety. In this study, we determined the prevalence and incidence of antimicrobial resistance in $V$. parahaemolyticus in the southern Fujian coast, China. A total of 62 isolates were confirmed in retail aquatic products from June to October of 2018. The serotype 03:K6 strains, the virulence genes $t d h$ and $t r h$, antibiotic susceptibility and molecular typing were investigated. Then plasmid profiling analysis and curing experiment were performed for multidrug-resistant strains. The results showed that the total occurrence of $V$. parahaemolyticus was $31 \%$ out of 200 samples. Five strains $(8.1 \%)$ out of 62 isolates were identified as the V. parahaemolyticus 03:K6 pandemic clone. A large majority of isolates exhibited higher resistance to penicillin $(77.4 \%)$, oxacillin $(71 \%)$, ampicillin $(66.1 \%)$ and vancomycin (59.7\%). Seventy-one percent $(44 / 62)$ of the isolates exhibited multiple antimicrobial resistance. All 62 isolates were grouped into 7 clusters by randomly amplified polymorphic DNA, and most of the isolates $(\mathbf{8 0 . 6 \% )}$ ) were distributed within cluster A. Plasmids were detected in approximately $75 \%$ of the isolates, and seven different profiles were observed. Seventy-six percent $(25 / 33)$ of the isolates carrying the plasmids were eliminated by $0.006 \%$ SDS incubated at $42^{\circ} \mathrm{C}$, a sublethal condition. The occurrence of multidrug-resistant strains could be an indication of the excessive use of antibiotics in aquaculture farming. The rational use of antimicrobial agents and the surveillance of antibiotic administration may reduce the acquisition of resistance by microorganisms in aquatic ecosystems.
\end{abstract}

Keywords: Multiple resistance, antimicrobial resistance genes, randomly amplified polymorphic DNA (RAPD), plasmid profiles

Received: January 3, 2020 Accepted: February 24, 2020

First published online: February 27, 2020

*Corresponding author Phone: +86-596-2528735 Fax: +86-596-2528735 E-mail:53066681@qq.com

pISSN 1017-7825 eISSN 1738-8872

Copyright(C) 2020 by The Korean Society for Microbiology and Biotechnology

\section{Introduction}

Vibrio parahaemolyticus is a notorious marine organism that occurs naturally in estuaries and coastal regions throughout the world [1,2]. Normally, V.parahaemolyticus bioaccumulates in the guts of filter-feeding molluscan shellfish, and it is a leading cause of seafood-associated gastroenteritis through the consumption of raw or undercooked aquatic foods [3, 4]. Antibiotics abuse has given rise to the global development and spread of drug resistance $[1,5,6]$. Clearly, $V$. parahaemolyticus can be a reservoir of resistance-associated genes and may play a role in the evolution and spread of antimicrobial resistance (AMR) in aquatic environments [1,7].

AMR is a critical public health issue in coastal regions [1]. Antibiotics have been used in food animals for treatment, disease prevention, or growth promotion, and they have accelerated the spread of resistant bacteria and resistance genes from food animals to humans along the food chain $[1,5]$. Furthermore, antimicrobial agents are released into marine aquaculture environments by agricultural runoff, providing opportunities for $V$. parahaemolyticus to acquire resistance mechanisms and subsequently concentrate in filter-feeding shellfish [6]. Unfortunately, the excessive and inappropriate use of antibiotics has resulted in the emergence of bacterial resistance to regular prescribed antibiotics, which can lead to failures in the available treatment options for common infections [8].

Molecular typing is important for assessing the transmission and diversity of strains and for differentiating between new infections and relapses [9]. All sorts of molecular typing tools have been used to study the prevalence of V.parahaemolyticus, and these methods include multi-locus sequence typing (MLST) $[8,10]$, pulsed-field gel 
electrophoresis (PFGE) [11], repetitive extragenic palindromic element-polymerase chain reaction (REP-PCR) [12], enterobacterial repetitive intergenic consensus (ERIC) $[8,13]$ and random amplified polymorphic DNA (RAPD) [14], etc. RAPD is useful for epidemiological studies because it provides adequate information on the source of infection, routes of transmission, suitable control measures, molecular markers of virulent and hostspecific strains, and genetic relatedness of different bacterial isolates $[1,14,15]$. Moreover, RAPD is easier to perform, labor-saving, faster, highly reproducible and requires significantly less special equipment and reagents [16] compared with other methods, such as PFGE. RAPD can be used in molecular subtyping for $V$. parahaemolyticus independently or in combination with other typing methods [16, 17]. The RAPD profiles were determined for the 62 isolates of V.parahaemolyticus from the southern Fujian coast, and the relationship between drug resistance and genetic diversity was studied in this paper.

The coastal area of Fujian is important for the production and processing of aquatic products. In recent years, the $V$. parahaemolyticus epidemic has been more serious in this region, especially in summer and autumn. In aquaculture, the excessive use of antibiotic drugs is very common, and it causes the more serious problem of drug resistance in $V$. parahaemolyticus. To our knowledge, no similar studies have been reported along the southern Fujian coast, an important aquaculture area in China. In this paper, 62 strains of V. parahaemolyticus that were isolated from aquatic products were studied by molecular typing, drug resistance gene analysis, drug resistance observation and a clearance test for drug resistance plasmids to understand the resistance status of $V$. parahaemolyticus.

\section{Materials and Methods \\ Bacterial Strains}

From June to October of 2018, a total of 200 aquatic product samples were obtained, namely short-necked clam (Paphia variegata, $n=42$ ), Asiatic hard clam (Meretrix meretrix, $n=36$ ), razor clam (Sinonovacula constricta, $n=34$ ), oyster (Crassostrea plicatula, $n=45$ ) and white shrimp (Penaeus vannamei, $n=43$ ), which were collected from retail markets along the southern Fujian coast in China. The first three species are widely cultured in the southeastern coastal provinces of China, while the last two are grown along the southern Fujian coast. Suspected colonies were screened on thiosulfate-citrate-bile salts-sucrose (TCBS, Beijing Land Bridge Technology Co., Ltd., China) agar plates. Presumptive isolates of $V$. parahaemolyticus were confirmed by the presence of the speciesspecific gene blaCARB-17 [18] and toxR [19] genes using duplex PCR. The presumptive V. parahaemolyticus isolates were evaluated for the virulence genes $t d h$ and trh using duplex PCR [20]. The reaction mixture for PCR assay was performed in $25 \mu \mathrm{l}$, which contained $12.5 \mu \mathrm{l}$ of $2 \times$ Taq Master Mix (Shanghai Generay Biotech Co., Ltd., China), $1 \mu \mathrm{M}$ of each primer, $3 \mu \mathrm{l}$ of DNA template and the remaining volume of double-distilled water. The thermal-cycling program is as follows: initial denaturation at $95^{\circ} \mathrm{C}$ for $5 \mathrm{~min} ; 30$ cycles of $95^{\circ} \mathrm{C}$ for $30 \mathrm{sec}$, an annealing temperature of $58^{\circ} \mathrm{C}$ for $30 \mathrm{sec}$, and $72^{\circ} \mathrm{C}$ for $30 \mathrm{sec}$ with a final extension of $72^{\circ} \mathrm{C}$ for $5 \mathrm{~min}$. These positive strains were stored at $-80^{\circ} \mathrm{C}$ for further analysis. The population numbers of $V$. parahaemolyticus in each aquatic sample were determined using the most probable number (MPN) method according to the Bacteriological Analytical Manual standard method and a previous study [4, 13, 21].

\section{Screening of Serovar O3:K6 by Triplex PCR}

Triplex PCR was performed to detect the toxR gene specific to the Vibrio species, the serogroup O3-specific gene $v p 0209$ and the serovar K6-specific gene $v p 0230$ according to Zhang's protocol [22-24]. The forward (F) and reverse (R) primers of the three detected genes are listed in Table 1. These oligonucleotide primers were synthesized by Sangon Biotech (China). The amplification conditions used for the triplex PCR were set up according to the protocol of Zhang et al. with slight modifications [24]. In brief, the $25 \mu$ reaction mixture was composed of $12.5 \mu \mathrm{l}$ of $2 \times$ Taq Master Mix (Shanghai Generay Biotech Co., Ltd.), each primer at $0.5 \mu \mathrm{M}, 6.5 \mu \mathrm{L}$ of $\mathrm{ddH}_{2} \mathrm{O}$, and $2 \mu \mathrm{l}$ of DNA template. The thermal cycling parameters for PCR were as follows: denaturation at $95^{\circ} \mathrm{C}$ for $2 \mathrm{~min}, 30$ cycles of $95^{\circ} \mathrm{C}$ for $30 \mathrm{sec}$, an annealing temperature of $56^{\circ} \mathrm{C}$ for $30 \mathrm{sec}$, and $72^{\circ} \mathrm{C}$ for $1 \mathrm{~min}$, with a final extension of $72^{\circ} \mathrm{C}$ for $5 \mathrm{~min}$. PCR was performed on a Bio-Rad PTC-200 Thermal Cycler (Bio-Rad, USA). The amplified products were analyzed electrophoretically on a 1.2\% agarose gel containing Gel Stain (Sangon Biotech, China) and photographed using an Amersham Imager 600 UV (GE Healthcare, USA).

\section{RAPD-PCR Genotyping}

The randomly designed 9-mer oligonucleotide primer P1 (5'-AAGAGCCGT-3') was used for RAPD typing as described previously by Hara-Kudo et al. [25]. The reactions were performed in $25 \mu$ l volumes consisting of $2.5 \mu \mathrm{l}$ of $10 \times$ PCR buffer (Shanghai Generay Biotech Co., Ltd.), $1 \mu \mathrm{l}$ of primer $(50 \mathrm{pmol} / \mu \mathrm{l}), 2 \mu \mathrm{l}$ of purified genomic DNA ( $50 \mathrm{ng} / \mu \mathrm{l}), 0.2 \mu \mathrm{l}$ of the $10 \mathrm{mM}$ dNTPs, $0.5 \mu \mathrm{l}$ of Taq DNA polymerase $(5 \mathrm{U} / \mu \mathrm{l})$ and $18.8 \mu \mathrm{l}$ of sterile distilled water. The following conditions were used for the amplification: initial denaturation at $94^{\circ} \mathrm{C}$ for $3 \mathrm{~min}, 40 \mathrm{cycles}$ of denaturation at $94^{\circ} \mathrm{C}$ for $1 \mathrm{~min}$, primer annealing at $36^{\circ} \mathrm{C}$ for $1 \mathrm{~min}$ and extension at $72^{\circ} \mathrm{C}$ for $2 \mathrm{~min}$, with a final extension at $72^{\circ} \mathrm{C}$ for $7 \mathrm{~min}$. The RAPD products were visualized using the same method as described above. A gel imaging map was analyzed using Gel Pro Analyzer 4.5 software, and the bands were marked as 0 (absence) or 1 (presence). Cluster analyses of RAPD data were performed using the unweighed pair group method of arithmetic averages (UPGMA) and NTSYS-pc (Version 2.10, http://www.exetersoftware.com/cat/ntsyspc.html).

\section{Antimicrobial Susceptibility Testing}

The antibiotic susceptibility of the 62 isolates was tested using the disk diffusion method on Mueller-Hinton agar (MHA) (OXOID Limited, China) in accordance with the guidelines by the Clinical and Laboratory Standards 
Table 1. Oligonucleotide primers used in this study.

\begin{tabular}{|c|c|c|c|c|}
\hline Target genes & Primer & Sequence ( $5^{\prime}$ to $\left.3^{\prime}\right)$ & $\begin{array}{l}\text { Amplicon } \\
\text { size (bp) }\end{array}$ & $\begin{array}{c}\text { Annealing } \\
\text { temperature } \\
\left({ }^{\circ} \mathrm{C}\right)\end{array}$ \\
\hline \multirow[t]{2}{*}{ blaCARB-17 } & CARB-F & ACC(T)TTGATGGAAGATA & 303 & 58 \\
\hline & CARB-R & T(C)TAACTTTCTTTGTAGTGC(A) & & \\
\hline \multirow[t]{2}{*}{ tox $R$} & toxR-F & GTCTTCTGACGCAATCGTTG & 368 & 58 \\
\hline & toxR-R & ATACGAGTGGTTGCTGTCATG & & \\
\hline \multirow[t]{2}{*}{$t d h$} & tdh-F & GTAAAGGTCTCTGACTTTTGGAC & 269 & 58 \\
\hline & tdh-R & TGGAATAGAACCTTCATCTTCACC & & \\
\hline \multirow[t]{2}{*}{ trh } & trh-F & TTGGCTTCGATATTTTCAGTATCT & 500 & 58 \\
\hline & trh-R & CATAACAAACATATGCCCATTTCCG & & \\
\hline RAPD & random site & AAGAGCCGT & Random size & 36 \\
\hline \multirow[t]{2}{*}{ blaTEM } & blaTEM-F & ATAAAATTCTTGAAGACGAAA & 1086 & 58 \\
\hline & blaTEM-R & GACAGTTACCAATGCTTAATC & & \\
\hline \multirow[t]{2}{*}{ blaOXA } & blaOXA-F & ACACAATACATATCAACTTCGC & 885 & 60 \\
\hline & blaOXA-R & AGTGTGTTTAGAATGGTGATC & & \\
\hline \multirow[t]{2}{*}{ blaSHV } & blaSHV-F & GCGAAAGCCAGCTGTCGGGC & 304 & 58 \\
\hline & blaSHV-R & GATTGGCGGCGCTGTTATCGC & & \\
\hline \multirow[t]{2}{*}{ blaCTX-M } & blaCTX-M-F & GTGCAGTACCAGTAAAGTTATGG & 538 & 58 \\
\hline & blaCTX-M-R & CGCAATATCATTGGTGGTGCC & & \\
\hline \multirow[t]{2}{*}{ strA } & strA-F & CCTGGTGATAACGGCAATTC & 546 & 58 \\
\hline & strA-R & CCAATCGCAGATAGAAGGC & & \\
\hline \multirow[t]{2}{*}{$s t r B$} & strB-F & ATCGTCAAGGGATTGAAACC & 509 & 54 \\
\hline & strB-R & GGATCGTAGAACATATTGGC & & \\
\hline \multirow[t]{2}{*}{ tet $A$} & tetA-F & GCTACATCCTGCTTGCCTTC & 210 & 46 \\
\hline & tetA-R & CATAGATCGCCGTGAAGAGG & & \\
\hline \multirow[t]{2}{*}{ tet $B$} & tet B-F & TTGGTTAGGGGCAAGTTTTG & 659 & 55 \\
\hline & tetB-R & GTAATGGGCCAATAACACCG & & \\
\hline \multirow[t]{2}{*}{ tetM } & tetM-F & GTRAYGAACTTTACCGAATC & 633 & 52 \\
\hline & tetM-R & ATCGYAGAAGCGGRTCAC & & \\
\hline catI & $\mathrm{C}-1$ & GGTGATATGGGATAGTGTT & 349 & 57.5 \\
\hline catII & $\mathrm{C}-2$ & GATTGACCTGAATACCTGGAA & 567 & 54 \\
\hline catIII & C-3 & CCATACTCATCCGATATTGA & 275 & 54 \\
\hline catIV & C-4 & CCGGTAAAGCGAAATTGTAT & 451 & 60.5 \\
\hline Cat reverse universal primer & $\mathrm{C}-\mathrm{R}$ & CCATCACATACTGCATGATG & - & - \\
\hline \multirow[t]{2}{*}{ gyrA } & gyrA-F & CGATTGGAACAAACCATATAAA & 200 & 56 \\
\hline & gyrA-R & CGGTGTAACGCATTGCCGCA & & \\
\hline \multirow[t]{2}{*}{$\operatorname{parC}$} & parC-F & CTTGGTCTTTCGGCATCAGC & 214 & 56 \\
\hline & parC-R & CTTCGGTATAACGCATTGCC & & \\
\hline \multirow[t]{2}{*}{ intI1 } & IntI1-F & GGGTCAAGGATCTGGATTTCG & 483 & 58 \\
\hline & IntI1-R & ACATGCGTGTAAATCATCGTCG & & \\
\hline \multirow[t]{2}{*}{ intI2 } & IntI2-F & CACGGATATGCGACAAAAAGGT & 788 & 60.8 \\
\hline & IntI2-R & GTAGCAAACGAGTGACGAAATG & & \\
\hline \multirow[t]{2}{*}{ intI3 } & IntI3-F & GCCTCCGGCAGCGACTTTCAG & 979 & 60.8 \\
\hline & IntI3-R & ACGGATCTGCCAAACCTGACT & & \\
\hline \multirow[t]{2}{*}{ Gene cassette } & Hep58 & TCATGGCTTGTTATGACTGT & Random size & 45 \\
\hline & Hep59 & GTAGGGCTTATTATGCACGC & & \\
\hline \multirow[t]{2}{*}{$v p 0230$} & VP-0230F & TCCTGTTGTGATAAAGTTGGCATT & 623 & 56 \\
\hline & VP-0230R & CCGAATCAAGAACTAACCCACA & & \\
\hline \multirow[t]{2}{*}{ vp0209 } & VP-0209F & GCATCAACCCATTTCAACTT & 240 & 56 \\
\hline & VP-0209R & TTCCATACTTGGGTTGAGTTTTC & & \\
\hline
\end{tabular}

Institute (CLSI, 2015). Thirty selected antimicrobials belonging to 11 classes used in this study were as follows: $\beta$ lactam (piperacillin: TZP, carbenicillin: CAR, oxacillin: OX, ampicillin: AMP, piperacillin: PRL, ceftazidime: CAZ, cefradine: CE, cephalexin: CL, cefazolin: KZ, cefuroxime: CXM, cefoperazone: SCF, cefatriaxone: CRO), aminoglycoside (gentamicin: CN, kanamycin: K, amikacin: AM, neomycin: N), macrolide (erythromycin: E, maddie mycin: MAG), tetracycline (tetracycline: TET, minocycline: $\mathrm{MH}$, doxycycline: $\mathrm{DO}$ ), quinolone (ciprofloxacin: CIP, norfloxacin: NOR, ofloxacin: OFLX), glycopeptide (vancomycin: VA), chloramphenicol (chloramphenicol: C), nitrofurans (furazolidone: FT), sulfonamides (trimethoprim-sulfamethoxazole: SXT), polypeptide (polymyxin B: $\mathrm{PB}$ ), and lincosamides (clindamycin: DA). The results were expressed as sensitive (S), intermediate (I), or resistant (R) according to the method by the Clinical and Laboratory Standards Institute (CLSI, 2015). Escherichia coli ATCC 25922 was used as the reference strain for the antimicrobial susceptibility detection. The antimicrobial resistance of all the isolates was performed with three repetitions. 


\section{MAR Index and Multidrug-Resistant (MDR) Definition}

The MAR index was created by Krumperman (1983), and it was calculated using the formula a/b where 'a' represents the number of antibiotics to which the specific isolate is drug-resistant, and 'b' represents the total number of multiple antibiotics to which the specific isolate has been exposed [26]. MDR was defined as the nonsusceptibility of the organism to at least one agent in three or more categories of antimicrobials. In our study, the MAR and MDR were calculated according to the above definition [26].

\section{Evaluation of Antibiotic Resistance-Encoding Genes}

The 19 genes with antibiotic resistance to 7 classes of antibiotics were analyzed by PCR method. The 19 antibiotic resistance genes were associated with $\beta$-lactam (blaTEM, blaOXA, blaSHV, and blaCTX-M), Aminoglycoside (strA and $\operatorname{str} B$ ), Tetracycline (tet $A$, tet $B$, and $t e t M)$, Chloramphenicol (catI, catII, cat III, and cat IV), Quinolone resistant determinant region ( $g y r A$ and parC), integron (intI1, intI2, and intI3) and the class I integron variable region gene box. The primers for the antibiotic resistance genes and the PCR conditions are shown in Table 1. The amplified products were analyzed using the same method as described above.

\section{Plasmid Profiling Analysis}

The multiple drug resistant strains were evaluated for the presence of plasmids. Plasmid extraction was performed using the centrifugal column type of plasmid preparation kit method (Generay Biotech.). Electrophoretic separation was performed at $80 \mathrm{~V}$ for $50 \mathrm{~min}$ and a molecular weight marker DL2000 (Bioteke, China) was included. The gels were visualized under a UV transilluminator and recorded as TIFF files using a Gel Documentation System (GelDoc EZ imager, Bio-Rad).

\section{Plasmid Curing Experiments}

The plasmids were cured for the isolates that were resistant to more than one antibiotic. The protocol was performed using Luria-Bertani broth supplemented with $3 \% \mathrm{NaCl}$ and $0.006 \%$ sodium dodecyl sulfate (SDS) at a $42^{\circ} \mathrm{C}$ sublethal condition [27]. The plasmid DNA were extracted using a TIANprep Mini Plasmid Kit II (TIANGEN BIOTECH CO., LTD China). The plasmid pattern analysis was visualized using the same method as described above. After the plasmid was successfully cleared, the resistance of these strains was tested again by disk diffusion method.

\section{Results}

Prevalence and Density of V.parahaemolyticus in Aquatic Products

$V$. parahaemolyticus is one of the major food-borne bacteria and is frequently isolated from various aquatic products. From June to October 2018, we tested 200 aquatic food samples from 10 retail markets along the southern Fujian coast. The prevalence of V. parahaemolyticus in different samples is shown in Table 2. Overall, $V$. parahaemolyticus was detected in $76(38 \%)$ of the 200 samples identified by traditional microbiological method, including the appearance of green colonies on TCBS agar and microscopic examination (gram-negative, curved rod-shaped, nonspore-forming bacilli), which are consistent with the national food safety standard of microbiological examination of food V. parahaemolyticus (GB4789.7-2013). Using duplex PCR for the speciesspecific toxR and blaCARB-17 genes, 62 of the 200 samples (31\%) were determined to be positive for the presence of $V$. parahaemolyticus. The target bands of $303 \mathrm{bp}$ (blaCARB-17) and $368 \mathrm{bp}$ (toxR) were obtained by PCR amplification and gel electrophoresis (Fig. 1). All 62 isolates showed the normal biochemical characteristics of $V$. parahaemolyticus. These 62 isolates were the sum of $9(21 \%), 7(19 \%), 12(35 \%), 29(64 \%)$, and $5(12 \%)$ isolates as collected from the short-necked clam, Asiatic hard clam, razor clam, oyster and white shrimp, respectively (Table 2). Three isolates containing the $t d h$ gene were obtained from oyster samples, and no isolates with the trh gene were found. In our study, the pandemic clone O3:K6 serotype was determined by triplex PCR method. Five isolates were identified as strains of serovar O3:K6, which carry the specific genes $v p 0209, v p 0230$, and toxR (Fig. 2). The densities were $<10 \mathrm{MPN} / \mathrm{g}$ in $61.3 \%$ (38/62) of the V. parahaemolyticus isolates. However, the densities of the remaining positive samples (24/62) ranged between 10 and $100 \mathrm{MPN} / \mathrm{g}$ (Table 2). According to the national food safety standard limit of pathogenic bacteria in food (GB29921-2013), these results of MPN are the acceptable level of pathogenic bacteria in China.

Table 2. Prevalence of V. parahaemolyticus in different samples collected in southern Fujian coast, China.

\begin{tabular}{lccccc}
\hline Sample category & $\begin{array}{c}\text { Number of } \\
\text { samples collected }\end{array}$ & $\begin{array}{c}\text { Positive } \\
\text { rate }\end{array}$ & $\begin{array}{c}\text { Positive } \\
\text { number }\end{array}$ & \multicolumn{2}{c}{$\begin{array}{c}\text { Number of samples containing V.parahaemolyticus } \\
\text { (MPN/g) }\end{array}$} \\
\hline Short-necked clam & 42 & $21 \%$ & 9 & $<10$ & 4 \\
Asiatic hard clam & 36 & $19 \%$ & 7 & 5 & 3 \\
Razor clam & 34 & $35 \%$ & 12 & 4 & 5 \\
Oyster & 45 & $64 \%$ & 29 & 19 & 10 \\
White shrimp & 43 & $12 \%$ & 5 & 3 & 2 \\
Total & 200 & $31 \%$ & 62 & 38 & 24 \\
\hline
\end{tabular}




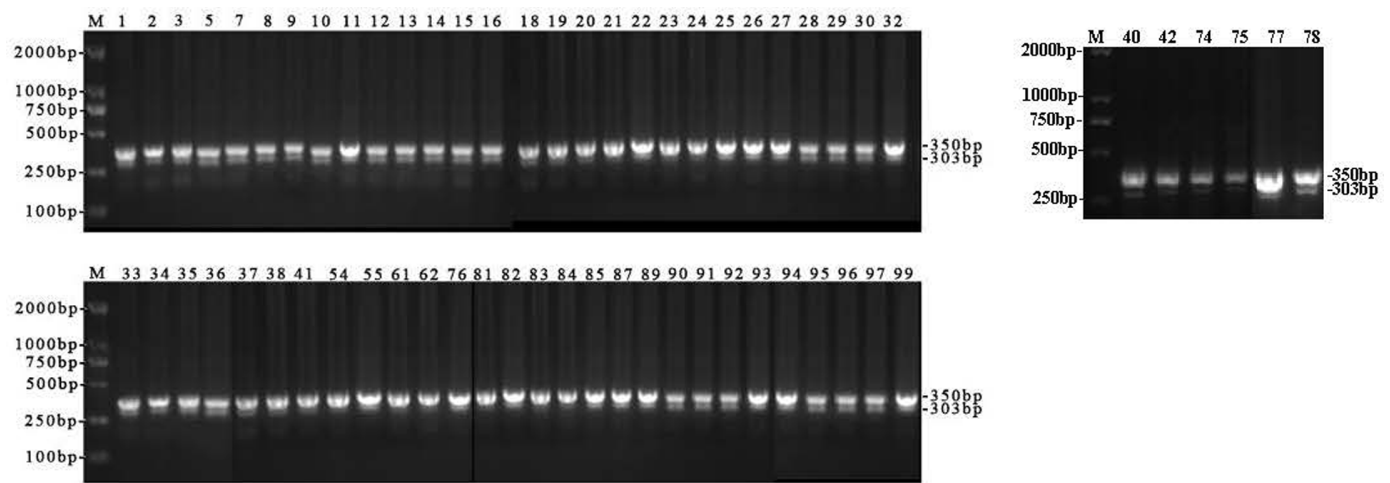

Fig. 1. Agarose gel (1.2\%) electrophoresis showing the results of duplex PCR amplification for the blaCARB17 and toxR genes of $62 \mathrm{~V}$. parahaemolyticus isolates. The amplified bands show the expected molecular size of $303 \mathrm{bp}$ for the blaCARB-17 gene and 368 bp for the toxR gene. M: DNA size markers; lanes with arabic numbers are the isolates of $V$. parahaemolyticus.

\section{RAPD Typing}

In this study, a total of 62 isolates of $V$. parahaemolyticus were analyzed by RAPD to estimate their genetic relationship. The results of RAPD fingerprinting showed strong genetic diversity (Fig. 3). The DNA profiles from the RAPD exhibited 3-10 amplified bands ranging from $180 \mathrm{bp}$ to approximately 4,000 bp. After hierarchical clustering analysis with the UPGMA, these polymorphic patterns were divided into 7 clusters (designated as A$\mathrm{G})$. Most isolates $(80.6 \%, 50 / 62)$ were distributed in cluster A. There is only one strain in three clusters known as D (62\#), $\mathrm{E}(85 \#)$ and $\mathrm{G}(36 \#)$. The three isolates (25\#, 83\#, and 87\#) were distributed within the B cluster. The four isolates (89\#, 90\#, 91\#, and 94\#) were distributed within the C cluster. Two isolates (81\# and 82\#) belonged in cluster $\mathrm{F}$. The results show that the typing is correlated with the geographical distribution of the samples. The isolates from the white shrimp samples collected from Yunxiao were primarily grouped into B, E, F, and G. Most of the V. parahaemolyticus isolated from Zhangzhou city were grouped into cluster A, but the other 4 strains were grouped into clusters B and C. One strain of white shrimp from Longhai was grouped into cluster D only. The strains isolated from Dongshan were not grouped into the same cluster as those from the closer location of Yunxiao. Instead, these strains were grouped into cluster A with those isolated from Zhangzhou City.

\section{Antimicrobial Susceptibility}

Thirty antimicrobials, including $\beta$-lactams, quinolones, aminoglycosides, tetracyclines, macrolides, lincosamides, chloramphenicols, sulfonamides, glycopeptides and polymyxins, were chosen for antimicrobial susceptibility testing, and the susceptible, intermediate and resistance rates are shown in Table 3 . The antimicrobial susceptibility testing ranges are also presented. None of the 62 isolates were resistant to CAZ, SCF,

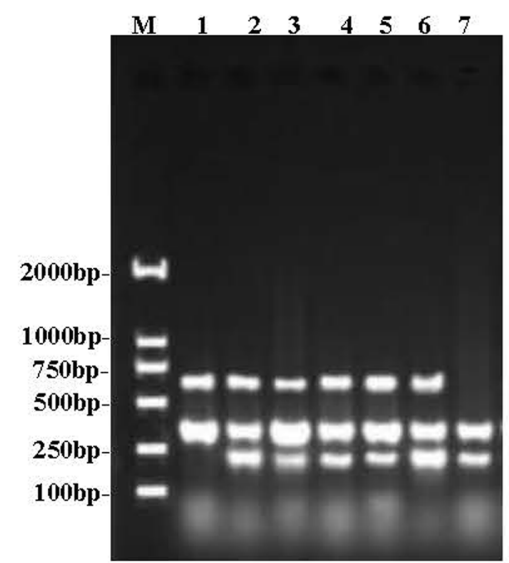

Fig. 2. Agarose gel (1.2\%) electrophoresis showing the results of triplex PCR amplification for screening of the 03:K6 serovar in $62 \mathrm{~V}$. parahaemolyticus isolates. Amplified bands with the expected molecular size of $240 \mathrm{bp}$ for the $v p 0209$ gene, $368 \mathrm{bp}$ for the toxR gene and $623 \mathrm{bp}$ for the $v p 0230$ gene. The $\mathrm{O} 3$ serovar was identified during the detection of the $v p 0209$ and toxR genes. The K6 serovar was identified when detecting the $v p 0230$ and toxR genes. Strains of serotype O3:K6 were confirmed when the 3 genes were detected simultaneously. Lane M, DL 2000 DNA marker; Lane 1, K6 serotype strain; Lane 7, O3 serotype strain; Lane 2-6, O3:K6 serotype isolates. 


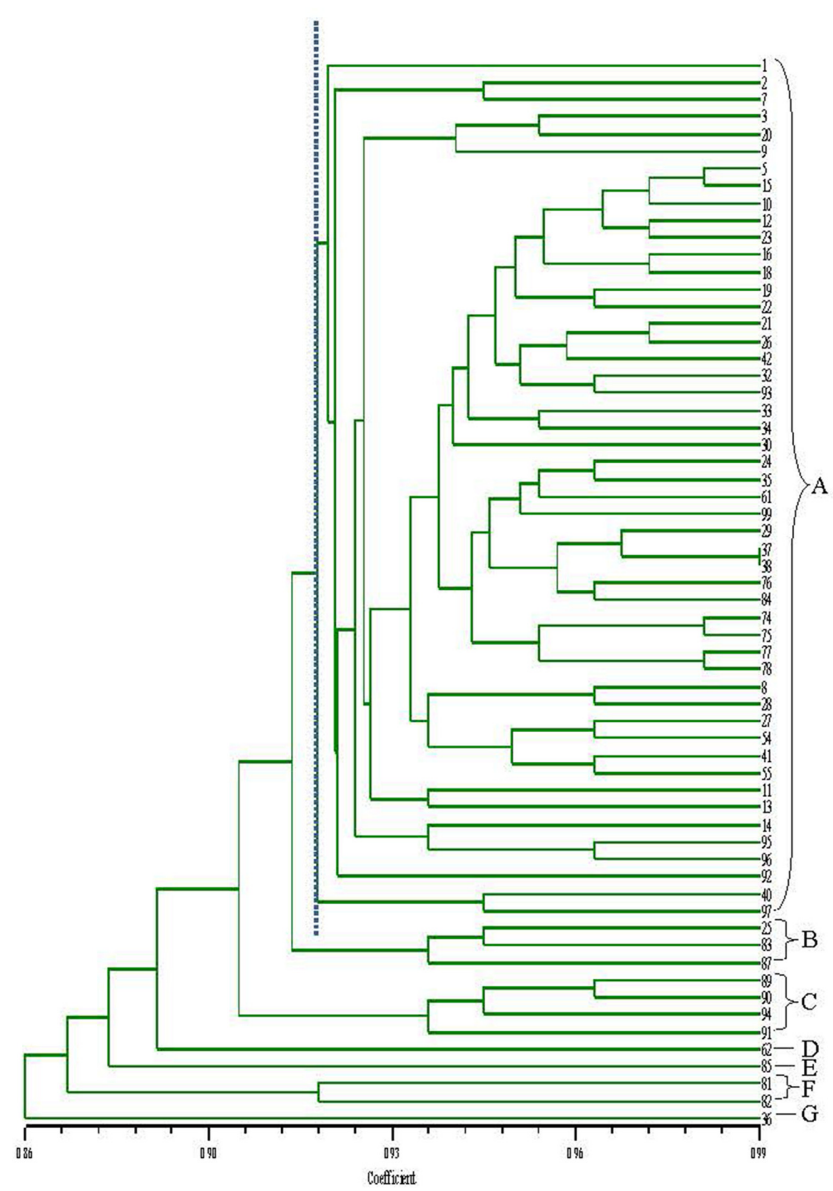

Resistance profile

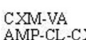

AMP-CL-CXM-PRL-VA

CN-CXM-OX-PRL-VA

CXM-VA
CXM-K.OX-PRL-VA MP-DO-OX-VA

CMP-CXM-OX-PRL-VA

CXM-VA
AMP-C-PRL-VA

AMP-VA-PRL-VA

CXM-VA

AMP-OX-PRL
AMP-OX-PRL-

AMP-OX-PRL-VA

PRL

AA

AMP-VA
AMP-CE-CL-OX-PRL

AMP-OX-PRL-V

CXM-VA
AMP-CXM-OX-PRL

NONE

AMP-CXM-DA-OX-PRL

AMP-CE-CL-OX-PRL-VA

CXM-VA
CXM-VA

CXM-VA MP-KZ-OX-PRL-TE-VA AMP-OX-PRL-VA OX-VA VA C-OX-PRL-VA M.C CXM-VA
AMP-CE-OX-PR AMP-CE-
NONE
CXM-VA AMP-OX-PRL-SXT-TE-VA CE-CL-OX-PRL-VA AMP.CE-CL AMP-CE-CXIM-OX-P
AMP-OX-PRL-VA AMP-OX-PRL AMP-OX-PRL AMP-CL-OX-PRL AMP-OX-PRL-VA AMP-OX-PRL
Resistance genes pattern

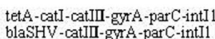
catI-catIII-gryA-parc-tetM
blaSHV-tetA-gyrA-parC-BOX blaSHV-tetA-gyrA-parC-BOX
blaC TXM-tetA-catt-catII-gyrA-parC catIII-gyM-A-parC tetA-gyrA-parC
blac TXM-catII-gyrA-parC-intI1 blaSHV-tetA -gyrA $p$ blac TMM-tiA-carC -cat1-gyrA-parC blaC TXM-catIII-gyrA-parC gyrA-parC-BOX
blaC TXM-catII-gyrA-parC blaC TXM-tetA-catt-catII-gyrA-parC catin-gysi-AparC blaC TXM-tetA-cattI-griA-parc
blaSHV-blacT XM-gyra-parC blaSHV-blacTX tetM-gytA-parC
tetA-cattII-gyrA-parC
catIII-graA-parC ceta-gyrA-parC att-catII-Eyre-parC blaSHV-catin-gya-parC blac TXM-strA-gyrA-parC
tetA-tetM-catII-gyrA-parC tetA-tetM-catII
gyrA-parc
blaSHV-parc

catI-catII-gyrA-parC-intI1 tetA-catt-grat-parC

aSHV-catIII-gyrA-par laC TXM-tetA-tetM-catIV-gyrA-parC-int11 blaC TXM-tetA-catIV-gyrA-parC-BOX

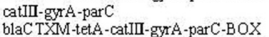
blac TXM-tetA-catII-gynA-parC-BOX
blac TXM-tetA-cat1-cat1I--gyrA-parC-int11

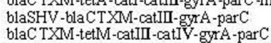
blaC TXM-tetM-catII-catIV-gynA-parC
blaSHV-tetA-catIII-gyrA-parC

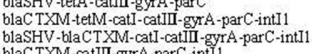
laC TXM-catIII-gyrA-parC-int blaC TXM-gyrA-parC letA-gya-parc blaC TXM-tetA-catt-catIII-catIV-gyrA-parC blac TXM-sta-gynA-par tetA-catII-gyrA-parC
tetA-tetM-catII-gyrA-parC-intI1 tetA-cat1-gyrA-parC-intI1 gyrA-parC
blaC TXM-strA-gyrA-parC blaC TXM-strA-gyrA-parC
tetA-gyrA-parC blaSHV-catIII-gyrA-parC

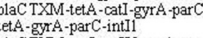

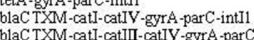

Fig. 3. The relationships the genetic diversity, resistance phenotype and genotype characterization of $62 \mathrm{~V}$. parahaemolyticus isolates. A phylogenetic tree of different V. parahaemolyticus isolates was drawn based on the RAPD results. A cluster analysis was performed using NTSYS-pc software based on the Dices similarity coefficient (SD) using a $1 \%$ position tolerance and the UPGMA.

CRO, TZP, CIP, NOR, OFLX, and E, and all the strains were sensitive to these drugs. The rates of resistance to AMP, OX, PRL, and VA reached $66.1 \%, 71 \%, 77.4 \%$, and $59.7 \%$, respectively. Smaller percentages of the isolates were resistant to CXM, CE, CL, CN, DO, TE, DA, C, and SXT, and the rates of resistance were $19.4 \%, 17.7 \%, 17.8 \%$, $4.8 \%, 8.1 \%, 6.5 \%, 8.1 \%, 11.3 \%$, and $6.5 \%$, respectively. By contrast, a large number of isolates had intermediate resistance to AM (67.7\%), CXM (80.6\%), CE (79\%), KZ (79\%), CL (82.3\%), MH (93.5\%), N (80.6\%), DO (91.9\%), and TE (72.6\%). All the isolates showed intermediate resistance to $\mathrm{K}$.

The MAR index was evaluated by finding the ratio of the number of antibiotics to which the organism was resistant to the total number of antibiotics used $[8,28]$. The MAR index, which was first proposed by Krumperman (1983), represents the extent of environmental contamination by antimicrobials, and it is used to assess the potential risk to human health. When an MAR index exceeds 0.2 , aquatic foods are considered as highrisk sources of antimicrobial contamination. In the present study, 71\% (44/62) of the V. parahaemolyticus isolates exhibited multiple antimicrobial resistance to at least 2 antimicrobials. The MAR index of most isolates was between 0.07 and 0.23 (Table 4). The maximum MAR index was attributed to one isolate that was resistant to 7 antibiotics. The present study found 11 out of $44(25 \%)$ multidrug-resistant strains of $V$. parahaemolyticus to be MDR. One of the serotype O3:K6 isolates was MDR. No correlation was found between the molecular typing and drug resistance spectrum of these strains.

\section{Antimicrobial Resistance Genotypes of V.parahaemolyticus}

Nineteen antimicrobial resistance genes belonging to 7 classes detected in $62 \mathrm{~V}$. parahaemolyticus isolates are shown in Table 5 and Fig. 3. The PCR method used to detect the occurrence of antimicrobial resistance genes (ARGs) was based on the genomic DNA of 62 isolates. Seven out of 19 resistance genes (blaTEM, blaOXA, strB, tet $B$, cat II, intI2, and intI3) were not detected in all the isolates. Other 12 genes were detected in one or more isolates. Clearly, all of the isolates carried two or more detected ARGs. Among these evaluated genes, parC was the most prevalent gene, with detection rate of $100 \%$, followed by gyrA, catIII, blaCTX-M, tetA, catI, intI1, blaSHV, 
Table 3. Results of antibiotic susceptibility tests on $62 \mathrm{~V}$.parahaemolyticus isolates from aquatic products.

\begin{tabular}{|c|c|c|c|c|c|c|c|}
\hline \multirow{2}{*}{ Antimicrobial class } & \multirow{2}{*}{ Antimicrobial agents } & \multicolumn{3}{|c|}{ V. parahaemolyticus $(n=62)$} & \multicolumn{3}{|c|}{ Zone diameters $(\mathrm{mm})$} \\
\hline & & No. (\%) of R & No. (\%) of I & No. (\%) of S & $\mathrm{R}$ & I & S \\
\hline \multirow[t]{13}{*}{$\beta$-lactams } & Amikaein (AM) & $0(0.0)$ & $42(67.7)$ & $20(32.3)$ & $\leq 13$ & $14-17$ & $\geq 18$ \\
\hline & Ampicillin (AMP) & $41(66.1)$ & $21(33.9)$ & $0(0.0)$ & $\leq 13$ & $14-16$ & $\geq 17$ \\
\hline & Carboxybenzicillin (CAR) & $0(0.0)$ & $24(38.7)$ & $38(61.3)$ & $\leq 14$ & $14-16$ & $\geq 17$ \\
\hline & Ceftazidime (CAZ) & $0(0.0)$ & $0(0.0)$ & $62(100.0)$ & $\leq 14$ & $15-17$ & $\geq 18$ \\
\hline & Cefoperazone (SCF) & $0(0.0)$ & $0(0.0)$ & $62(100.0)$ & $\leq 15$ & $16-20$ & $\geq 21$ \\
\hline & Cefatriaxone (CRO) & $0(0.0)$ & $0(0.0)$ & $62(100.0)$ & $\leq 15$ & $16-20$ & $\geq 21$ \\
\hline & Cefuroxime (CXM) & $12(19.4)$ & $50(80.6)$ & $0(0.0)$ & $\leq 14$ & $15-17$ & $\geq 18$ \\
\hline & Cefradine (CE) & $11(17.7)$ & $49(79.0)$ & $2(3.2)$ & $\leq 14$ & $15-17$ & $\geq 18$ \\
\hline & Cefazolin (KZ) & $0(0.0)$ & $49(79.0)$ & $13(21.0)$ & $\leq 14$ & $15-17$ & $\geq 18$ \\
\hline & Cephalexin (CL) & $11(17.8)$ & $51(82.3)$ & $0(0.0)$ & $\leq 14$ & $15-17$ & $\geq 18$ \\
\hline & Oxacillin (OX) & $44(71.0)$ & $18(29.0)$ & $0(0.0)$ & $\leq 10$ & $11-12$ & $\geq 13$ \\
\hline & Piperacillin (TZP) & $0(0.0)$ & $0(0.0)$ & $62(100.0)$ & $\leq 17$ & $18-20$ & $\geq 21$ \\
\hline & Penicillin (PRL) & $48(77.4)$ & $14(22.6)$ & $0(0.0)$ & $\leq 19$ & - & $\geq 20$ \\
\hline \multirow[t]{3}{*}{ Quinolones } & Ciprofloxacin (CIP) & $0(0.0)$ & $0(0.0)$ & $62(100.0)$ & $\leq 15$ & $16-20$ & $\geq 21$ \\
\hline & Norfloxacin (NOR) & $0(0.0)$ & $0(0.0)$ & $62(100.0)$ & $\leq 12$ & $13-16$ & $\geq 17$ \\
\hline & Ofloxacin (OFLX) & $0(0.0)$ & $0(0.0)$ & $62(100.0)$ & $\leq 12$ & $13-15$ & $\geq 16$ \\
\hline \multirow[t]{3}{*}{ Aminoglycoside } & Gentamicin $(\mathrm{CN})$ & $3(4.8)$ & $21(33.9)$ & $38(61.3)$ & $\leq 12$ & $13-14$ & $\geq 15$ \\
\hline & Kanamycin (K) & $0(0.0)$ & $62(100.0)$ & $0(0.0)$ & $\leq 13$ & $14-17$ & $\geq 18$ \\
\hline & Neomycin $(\mathrm{N})$ & $0(0.0)$ & $50(80.6)$ & $12(19.4)$ & $\leq 12$ & $13-16$ & $\geq 17$ \\
\hline \multirow[t]{3}{*}{ Tetracylines } & Doxycycline (DO) & $5(8.1)$ & 57 (91.9) & $0(0.0)$ & $\leq 12$ & $13-15$ & $\geq 16$ \\
\hline & Tetracycline (TET) & $4(6.5)$ & $45(72.6)$ & $13(21.0)$ & $\leq 14$ & $15-18$ & $\geq 19$ \\
\hline & Minocycline (MH) & $0(0.0)$ & $58(93.5)$ & $4(6.5)$ & $\leq 14$ & $15-18$ & $\geq 19$ \\
\hline \multirow[t]{2}{*}{ Macrolides } & Erythromycin (E) & $0(0.0)$ & $0(0.0)$ & $62(100.0)$ & $\leq 13$ & $14-22$ & $\geq 23$ \\
\hline & Medemycin (MAG) & $0(0.0)$ & $29(46.8)$ & $33(53.2)$ & $\leq 13$ & $14-17$ & $\geq 18$ \\
\hline Lincosamides & Clindamycin (DA) & $5(8.1)$ & $10(16.1)$ & $47(75.8)$ & $\leq 14$ & $15-20$ & $\geq 21$ \\
\hline Chloramphenicols & Chloramphenicol (C) & $7(11.3)$ & $0(0.0)$ & $55(88.7)$ & $\leq 12$ & $13-17$ & $\geq 18$ \\
\hline Sulfonamides & Chemitrim (SXT) & $4(6.5)$ & $4(6.5)$ & $54(87.1)$ & $\leq 12$ & $13-16$ & $\geq 17$ \\
\hline Glycopeptides & Vancomycin (VA) & $37(59.7)$ & $25(40.3)$ & $0(0.0)$ & $\leq 9$ & $10-11$ & $\geq 12$ \\
\hline Polymyxins & Polymyxin B (PB) & $0(0.0)$ & $19(30.6)$ & $43(69.4)$ & $\leq 8$ & $9-11$ & $\geq 12$ \\
\hline Nitrofurans & Furazolidone (FT) & $0(0.0)$ & $28(45.2)$ & $34(54.8)$ & $\leq 14$ & $15-16$ & $\geq 17$ \\
\hline
\end{tabular}

${ }^{\star} \mathrm{R}$, resistant; $\mathrm{I}$, intermediate resistance; $\mathrm{S}$, susceptibility.

tet $M$, cat IV, BOX and strA, and the detected percentages of them were $98.4 \%, 54 \%, 42.9 \%, 41.3 \%, 33.3 \%, 20.6 \%$, $17.5 \%, 11.1 \%, 11.1 \%, 9.5 \%$, and $5.8 \%$, respectively.

Plasmid Profiling and the Result of Plasmid Curing in the Isolates

Plasmid profiles of the 44 multiple drug resistant isolates were performed. Plasmids were found in 75\% (33/44) of the isolates and 7 different plasmid profiles (P1-P7) were observed (Table 6). No plasmid was detected in 11 isolates. Plasmid profiles $1(\mathrm{P} 1: 33 \mathrm{~kb})$ and $2(\mathrm{P} 2: 33 \mathrm{~kb}, 15 \mathrm{~kb})$ were the most common profiles among the isolates from aquatic products. The plasmids were eliminated by $0.006 \%$ SDS combined with a $42^{\circ} \mathrm{C}$ sublethal condition. Twenty-five of the 33 strains carrying the plasmid were not detected after the plasmid curing, and the elimination rate reached $76 \%$. The drug resistance of the strains from which the plasmids were cleared also changed. Isolates $11 \#$ and 82\# became moderately resistant to ampicillin, 20\# and 76\# became moderately resistant to cefuroxime, 14\# became susceptible to amikacin, 20\# became susceptible to kanamycin, 13\# became susceptible to oxacillin, and $82 \#$ became susceptible to penicillin from drug resistance.

\section{Discussion}

V. parahaemolyticus is considered as an important seafood-borne pathogen because of its involvement in outbreaks after the consumption of contaminated foods [29]. In recent decades, the antibiotics used in aquaculture have been largely used for therapeutic and prophylactic purposes, and antibiotic resistance is a global public health concern and a food safety problem [30]. In the present study, we analyzed 200 aquatic product specimens that were collected from the retail markets along the southern Fujian coast from June to October of 2018 and isolated $62 \mathrm{~V}$. parahaemolyticus strains, which had a positive rate of $31 \%$, suggesting the possibility that there were gastroenteritis cases associated with the consumption of uncooked seafood or cross-contaminated food in coastal areas during warmer months. Compared to other studies in China in recent years, this positive rate is slightly lower than the $47.2 \%$ reported for Jiangsu Province [14], the $38.0 \%$ reported in Zhejiang Province from 2010 to 2014 [31], the $43.75 \%$ observed from the markets of 11 cities in four provinces of South China [13] and the $34.3 \%$ reported in Shanghai from February 2014 to February 2015 [8]. Compared to other countries, our result is also significantly less than the $83 \%$ rate in raw oysters in Brazil [20], 67\% in fish in Jordan [19] and $80.8 \%$ in shrimp in Ecuador [32]. This finding indicates that the current status of V. parahaemolyticus prevalence in the southern Fujian coast is better than that of other regions. In our study, the positive rate of V.parahaemolyticus in oysters was 
Table 4. Multiple antimicrobial resistance (MAR) pattern of V.parahaemolyticus.

\begin{tabular}{|c|c|c|c|c|}
\hline Resistance pattern & Isolate & $\begin{array}{c}\text { Frequency of } \\
\text { occurrence }\end{array}$ & $\begin{array}{l}\text { Number of } \\
\text { antibiotics }\end{array}$ & $\begin{array}{l}\text { MAR } \\
\text { index }\end{array}$ \\
\hline $\mathrm{AM}, \mathrm{C}$ & 14 & 1 & 2 & 0.07 \\
\hline AMP, PRL & 33 & 1 & 2 & 0.07 \\
\hline AMP, VA & 23,34 & 2 & 2 & 0.07 \\
\hline CXM, VA & 1 & 1 & 2 & 0.07 \\
\hline OX, VA & 41 & 1 & 2 & 0.07 \\
\hline AMP, CE, CL & 87 & 1 & 3 & 0.1 \\
\hline AMP, CXM, VA & 11 & 1 & 3 & 0.1 \\
\hline OX, AMP, PRL & $22,82,91,93,94$ & 5 & 3 & 0.1 \\
\hline AMP, C, PRL, VA & 12 & 1 & 4 & 0.13 \\
\hline AMP, CE, OX, PRL & 92 & 1 & 4 & 0.13 \\
\hline AMP, CXM, OX, PRL & 29 & 1 & 4 & 0.13 \\
\hline AMP, CL, OX, PRL & 62,95 & 2 & 4 & 0.13 \\
\hline AMP, DO, OX, VA & 5 & 1 & 4 & 0.13 \\
\hline AMP, OX, PRL, VA & $16,21,26,35,54,61,85,90$ & 8 & 4 & 0.13 \\
\hline CE, CLVA, OX, PRL & 83 & 1 & 4 & 0.13 \\
\hline C, OX, PRL, VA & 13 & 1 & 4 & 0.13 \\
\hline AMP, CL, CXM, PRL, VA & 2 & 1 & 5 & 0.17 \\
\hline AMP, CE, CL, OX, PRL & 30,81 & 2 & 5 & 0.17 \\
\hline AMP, CE, CXM, OX, PRL & 89 & 1 & 5 & 0.17 \\
\hline AMP, CXM, DA, OX, PRL & 76 & 1 & 5 & 0.17 \\
\hline AMP, CXM, OX, PRL, VA & 15 & 1 & 5 & 0.17 \\
\hline AMP, OX, PRL, SXT, VA & 36 & 1 & 5 & 0.17 \\
\hline CN, CXM, OX, PRL, VA & 7 & 1 & 5 & 0.17 \\
\hline CXM, K, OX, PRL, VA & 20 & 1 & 5 & 0.17 \\
\hline AMP, CE, CL, OX, PRL, VA & 84 & 1 & 6 & 0.2 \\
\hline AMP, CXM, DO, OX, PRL, VA & 28 & 1 & 6 & 0.2 \\
\hline AMP, KZ, OX, PRL, TE, VA & 27 & 1 & 6 & 0.2 \\
\hline AMP, OX, PRL, SXT, TE, VA & 25 & 1 & 6 & 0.2 \\
\hline CL, CN, CXM, OX, PRL, VA & 9 & 1 & 6 & 0.2 \\
\hline AMP, C, CXM, DA, OX, PRL, VA & 8 & 1 & 7 & 0.23 \\
\hline
\end{tabular}

$64 \%$, which was significantly higher than that of other aquatic samples. Because oysters need to filter more seawater to provide essential nutrition by eating organic matter from the surrounding water, oysters simultaneously bioaccumulate more pathogenic organisms from water than other shellfish [8]. The positive isolates were confirmed by duplex PCR based on the detection of the blaCARB-17 and toxR genes, and ultimately, 62 out of $76 \mathrm{~V}$. parahaemolyticus strains were obtained. The above results are also consistent with the biochemical experiments and Gram staining test. Although many of the targeting genes (tlh and atpA) were used as species-

Table 5. Percentage of antimicrobial resistance genes for $V$. parahaemolyticus isolates $(n=62)$.

\begin{tabular}{lcc}
\hline Antimicrobial class & Antimicrobial resistance genes & Percentage of V.parahaemolyticus $(n=62)$ \\
\hline$\beta$-lactam & blaTEM & 0 \\
& blaOXA & 0 \\
& blaSHV & 17.5 \\
Quinolone & blaCTX-M & 42.9 \\
& gyrA & 98.4 \\
Aminoglycoside & parC & 100 \\
& strA & 5.8 \\
Tetracyline & strB & 0 \\
& tetA & 41.3 \\
& tetB & 0 \\
Chloramphenicol & tet $M$ & 11.1 \\
& catI & 33.3 \\
& catII & 0 \\
catIII & 54 \\
Integrase & catIV & 11.1 \\
& intI1 & 20.6 \\
I integron variable region & intI & 0 \\
\hline
\end{tabular}


Table 6. Plasmid pattern of V.parahaemolyticus from aquatic products.

\begin{tabular}{ll}
\multicolumn{1}{c}{ Plasmid pattern } & \multicolumn{1}{c}{ Isolates of V. parahaemolyticus } \\
\hline P1: $33 \mathrm{~kb}$ & $1,8,20,30,36,84,89,90,91,93,94,95$ \\
$\mathrm{P} 2: 33 \mathrm{~kb} ; 15 \mathrm{~kb}$ & $14,16,21,23,34,35,41,82,83,85,87,92$ \\
$\mathrm{P} 3: 33 \mathrm{~kb} ; 5 \mathrm{~kb}$ & 33 \\
$\mathrm{P} 4: 33 \mathrm{~kb} ; 18 \mathrm{~kb} ; 15 \mathrm{~kb}$ & $29,54,61,62,76$ \\
P5: $33 \mathrm{~kb} ; 15 \mathrm{~kb} ; 7 \mathrm{~kb} ; 1.5 \mathrm{~kb}$ & 11 \\
P6: $33 \mathrm{~kb} ; 18 \mathrm{~kb} ; 3 \mathrm{~kb} ; 1 \mathrm{~kb}$ & 22 \\
P7: $33 \mathrm{~kb} ; 15 \mathrm{~kb} ; 3 \mathrm{~kb} ; 0.75 \mathrm{~kb} ; 0.5 \mathrm{~kb}$ & 81 \\
None detected & $5,7,8,9,12,13,20,25,27,28,36$ \\
\hline
\end{tabular}

specific markers, some of them may share similar sequences within the Vibrio species, reducing the accuracy and specificity of these detection methods $[18,33]$. A novel blaCARB-17 gene was identified and found to be responsible for the intrinsic penicillin resistance in $V$. parahaemolyticus [18, 34]. Li et al analyzed the homologies of the tlh, atpA and blaCARB-17 genes, and the last is more highly conserved than the other two genes [18]. The specificity of the PCR based on the blaCARB-17 gene is $100 \%$, whereas the PCR targeting the th and atpA genes occasionally produced false positive results [18]. To improve the detection accuracy, a duplex PCR targeting the toxR and blaCARB-17 genes was developed and used to identify the $V$. parahaemolyticus in our study. Here, 3 $(4.8 \%)$ isolates were $t d h$-positive $V$. parahaemolyticus, whereas no trh-positive strains were found. The finding of fewer $t d h$-and/or trh-positive strains of $V$. parahaemolyticus indicates lower aquatic food risk. The pandemic O3:K6 serovars are considered possibly responsible for recent $V$. parahaemolyticus outbreaks in many countries, and the majority of infections are acquired by the means of polluted seafood $[35,36]$. In the present study, 5 isolates were identified as the serovar O3:K6 clone by triplex PCR method. These findings contrast with the results of a previous study $[37,38]$

The RAPD assay has been proved to be a powerful tool for discriminating among different species or subspecies and for the genetic analysis of phylogenetic relationships among strains or populations for a variety of microorganisms [14]. Yang et al assessed the genetic diversity among 251 isolates, and 33 different genetic patterns were grouped into 9 clusters at an $82 \%$ similarity level by RAPD method [39]. Chao et al identified 341 strains of $V$. parahaemolyticus, which were clustered into six molecular types and 23 subtypes using the RAPD analysis [14]. Zhao et al analyzed the genetic diversity of 157 isolates, and 73 different patterns were identified, which were grouped into 18 clusters [16]. Compared with pulsed-field gel electrophoresis (PFGE), enterobacterial repetitive intergenic consensus sequence PCR (ERIC-PCR) and repetitive extragenic palindromic sequence PCR (REPPCR), RAPD-PCR is superior in that it is completed more quickly, is cost-effective, less labor-intensive and is based on differences in nucleotide sequences in the whole genome [39]. The RAPD method has been used successfully for subdividing V. parahaemolyticus from different seafood in India and for other organisms [40-43]. Selecting effective primers is key to the successful implementation of RAPD. In reference to Chao et al 2009, we chose $\mathrm{P} 1$ as the amplification primer and obtained better RAPD patterns [14]. In this study, all 62 isolates were identified with individual patterns. These patterns were grouped into 7 RAPD clusters with a coefficient of more than 0.918 , and cluster A is predominantly present in the southern Fujian coast, China. These isolates (80.6\%) were grouped into the same RAPD cluster A but did not show identical RAPD patterns, suggesting that these isolates were genetically very closely related.

Vibrio is the most important pathogenic bacteria in aquaculture, and it often causes serious diseases and results in serious economic losses $[44,45]$. The global increase in the prevalence of antibiotic-resistant Vibrio is a major concern for food safety and fish farming [46]. Most of the tested antibiotics, such as ciprofloxacin, chloramphenicol, gentamicin and ceftazidime, are recommended as treatments for Vibrio infection [2, 3]. In the current study, $77.4 \%$ and $71 \%$ of the isolates showed resistance to penicillin and oxacillin, respectively, and these results were comparable to data obtained in other countries. Ceftazidime, cefoperazone, cefatriaxone, piperacillin, ciprofloxacin, norfloxacin, ofloxacin and erythromycin were found to be the most efficient at eliminating V. parahaemolyticus strains isolated from aquatic products along the southern Fujian coast of China (Table 3). The isolates in our collected samples were most sensitive to ceftazidime, cefoperazone, cefatriaxone, piperacillin, ciprofloxacin, norfloxacin, ofloxacin and erythromycin. This finding was satisfactory because these antibiotics are the preferred drugs for treating infections caused by Vibrio spp. [47]. The efficiency of ceftazidime, cefoperazone, cefatriaxone and piperacillin is related to their ability to inhibit the mucopeptide synthetase of the cell wall [48]. Thus, the synthesis of cell wall mucopeptides is blocked, the bacterial cell wall is defective, and the bacterial body expands and splits. To our knowledge, quinolones, tetracyclines and sulfonamides are widely used in coastal aquaculture in China. In our study, all 62 isolates were sensitive to ciprofloxacin, norfloxacin and ofloxacin. However, high incidences of intermediate resistance to minocycline (93.5\%) and furazolidone (45.2\%) were found. Similarly, most strains showed moderate tolerance to tetracyclines, including doxycycline and tetracycline, and the incidences of intermediate resistance are $91.9 \%$ and $72.6 \%$, respectively. Only a small number of strains showed resistance and moderate tolerance to sulfonamides, at $8.1 \%$ and $16.1 \%$, respectively. Notably, the results showed that $77.4 \%$ of the isolates were resistant to PRL. A higher percentage of antibiotic resistance was also found in AMP (66.1\%), OX (71\%) and VA (59.7\%). These results were partially consistent with other studies performed in China [6, 8, 49-51]. Surprisingly, isolates of $V$. parahaemolyticus were resistant to the firstgeneration cephalosporins (CE $17.7 \%$ and CL $17.8 \%$ ), but no resistance profile was observed within the isolates 
towards the third-generation cephalosporins (CAZ, SCF, and CRO). This finding is primarily due to the abuse of the first-generation cephalosporins over the past few decades. In summary, the strains that are resistant to quinolones, tetracyclines and sulfonamides are not particularly serious in this region. There is also a relatively high proportion of medium-resistant strains, and they may be transformed into drug-resistant strains under environmental stress. The high prevalence of antimicrobial resistance among seafood-derived $V$. parahaemolyticus is a risk to human health, and the resistant isolates might be a potential source of risk for the storage and transmission of resistance genes. This problem must be monitored by the relevant departments.

More than half $(71 \%)$ of the V. parahaemolyticus isolates demonstrated MAR to at least two antimicrobials (Table 4), with a MAR range of 0.07 to 0.23 and an average of 0.14 . The MAR index was as high as 0.23 in one isolate (8\#) that was resistant to seven antibiotics. The variation in the MAR index could be attributed to differences in the sources of samples, geographic distribution and test methodologies $[9,52]$. Among these factors, diverse geographical environments had differential selective pressures for the antibiotic resistance levels [9]. In Korea, high MAR indexes of 0.38 and 0.75 were found in V.parahaemolyticus strains from oyster samples [3]. In Malaysia, an MAR index of 0.93 was found in V.parahaemolyticus isolates from fish samples [44]. A high MAR index (0.63) was reported in V.parahaemolyticus isolates from environmental samples in England [53]. In this study, the MAR index of almost half of the isolates was less than 0.2, suggesting that most of the seafood presented lower risks of antimicrobial contamination in this region. V. parahaemolyticus lives in coastal and estuarine waters, which are particularly subject to environmental contamination by agricultural runoff or effluent from wastewater treatment plants. Aquatic bacteria suffer from selective pressure for antimicrobial resistance from the various levels of antimicrobials and heavy metals in the estuarine or offshore environments [54].

Among the 3 tetracycline resistance genes evaluated in our study, the detection frequencies of $\operatorname{tet} A$ and tet $M$ were approximately $41.3 \%$ and $11.1 \%$, respectively. The tet $A$ gene was found in Vibrio cholera isolates collected from shrimp ponds in Thailand [55]. Another tetracycline resistance gene, tet $B$, was not detected in this study. The aminoglycoside resistance gene strA was detected in $5.8 \%$ of the isolates and $\operatorname{str} B$ was not found. these results are not consistent with the findings by Lou et al. 2016 [2]. Two quinolone resistance genes evaluated here, gyrA and parC, were detected in $98.4 \%$ and $100 \%$ of the isolates, respectively, and they are normally detected in bacteria. Our findings demonstrated that isolates possessing resistance genes might not be resistant to the relevant antimicrobial agents. This study is the first large-scale survey on the antimicrobial resistance genes of seafoodderived V. parahaemolyticus isolates from the southern Fujian coast, China.

Plasmids are considered one of the most significant mediators of the speedy spread of antibiotic resistance within bacteria [56], so it is essential to screen genetic elements such as the plasmids associated with antibiotic resistance in microorganisms. In our study, we were able to detect the presence of plasmids within $75 \%$ of the isolates. Seven different plasmid patterns were shown. A $33 \mathrm{~kb}$ plasmid was found in all 44 multiple drug resistance isolates, indicating the presence of $30 \mathrm{~kb}$ plasmids in environmental Vibrio isolates, which is consistent with the findings of Zhang et al. [57]. As previously shown, bacterial antibiotic resistance profiles are associated with large plasmids and their conjugation [56]. In our study, no correlation was found between the antibiotic resistance patterns and the plasmid profiles.

In conclusion, we present a comprehensive study involving molecular typing, antimicrobial susceptibility, resistance-associated genotypes and plasmid profiles of $V$. parahaemolyticus isolated from aquatic products collected along the southern Fujian coast of China from June to October of 2018. The occurrence of $V$. parahaemolyticus was $31 \%$, which represents a decrease compared to other previous reports. The virulence gene $t d h$ was detected in three isolates (4.8\%), and the O3:K6 pandemic clone was determined in five strains (8.1\%). In addition, the results showed that $77.4 \%$ of the V.parahaemolyticus was resistant to PRL, and resistance was also shown to OX, AMP and VA. Notably, a large majority of isolates (71\%) exhibited multiple antimicrobial resistance. However, there is no apparent correlation among the resistance phenotype, genotype and plasmid patterns in our study. Given the complex situation of antimicrobial resistance in aquatic food, broader monitoring and investigation are needed in China.

\section{Acknowledgments}

This work was supported by the Project of Fujian Natural Science Foundation of China [grant number 2017J01453], the Class A Project of Fujian Provincial Education Department [grant number JA14203], the Zhangzhou Natural Science Foundation Project [grant number ZZ2016J20], the Open Project Program of Jiangsu Key Laboratory of Zoonosis [grant number R1402].

\section{Conflict of Interest}

The authors declare that they have no conflicts of interest.

\section{References}

1. Elmahdi S, Dasilva LV, Parveen S. 2016. Antibiotic resistance of Vibrio parahaemolyticus and Vibrio vulnificus in various countries: a review. Food Microbiol. 57: 128-134.

2. Lou Y, Liu HQ, Zhang ZH, Pan YJ, Zhao Y. 2016. Mismatch between antimicrobial resistance phenotype and genotype of pathogenic Vibrio parahaemolyticus isolated from seafood. Food Control 59: 207-211.

3. Kang CH, Shin YJ, Kim WR, Kim YG, Song KC, Oh EG, et al. 2016. Prevalence and antimicrobial susceptibility of Vibrio parahaemolyticus isolated from oysters in Korea. Environ. Sci. Pollut. R. 23: 918-926.

4. Xie TF, Xu XK, Wu QP, Zhang JM, Cheng JH. 2016. Prevalence, molecular characterization, and antibiotic susceptibility of Vibrio parahaemolyticus from ready-to-eat foods in China. Front. Microbiol. 7: 549. 
5. Odeyemi OA, Stratev D. 2016. Occurrence of antimicrobial resistant or pathogenic Vibrio parahaemolyticus in seafood. A mini review. Rev. Med. Vet. 67: 93-98.

6. He Y, Jin LL, Sun FJ, Hu QX, Chen LM. 2016. Antibiotic and heavy-metal resistance of Vibrio parahaemolyticus isolated from fresh shrimps in Shanghai fish markets, China. Environ. Sci. Pollut. Res. Int. 23: 15033-15040.

7. Li H, Tang R, Lou Y, Cui ZL, Chen WJ, Hong Q, et al. 2017. A comprehensive epidemiological research for clinical Vibrio parahaemolyticus in Shanghai. Front. Microbiol. 8: 1043.

8. Yu QQ, Niu MY, Yu MQ, Liu YH, Wang DP, Shi XM. 2016. Prevalence and antimicrobial susceptibility of Vibrio parahaemolyticus isolated from retail shellfish in Shanghai. Food Control 60: 263-268.

9. Ahmed HA, El RB, Hussein MA, Khedr M, Abo ER, Elashram A. 2018. Molecular characterization, antibiotic resistance pattern and biofilm formation of Vibrio parahaemolyticus and V. cholerae isolated from crustaceans and humans. Int. J. Food Microbiol. 274: 3137.

10. Han DS, Tang H, Ren CL, Wang GZ, Zhou L, Han CX. 2015. Prevalence and genetic diversity of clinical Vibrio parahaemolyticus isolates from China, revealed by multilocus sequence typing scheme. Front. Microbiol. 6: 291.

11. Lopatek M, Wieczorek K, Osek J. 2018. Antimicrobial resistance, virulence factors, and genetic profiles of Vibrio parahaemolyticus from seafood. Appl. Environ. Microbiol. 84: e00537-00518.

12. Paydar M, Teh CSJ, Thong KL. 2013. Prevalence and characterisation of potentially virulent Vibrio parahaemolyticus in seafood in Malaysia using conventional methods, PCR and REP-PCR. Food Control 32: 13-18.

13. Xie TF, Wu QP, Xu XK, Zhang JM, Guo WP. 2015. Prevalence and population analysis of Vibrio parahaemolyticus in aquatic products from South China markets. FEMS Microbiol. Lett. 362: fnv178.

14. Chao GX, Jiao XN, Zhou XH, Yang ZQ, Huang JL, Zhou LP, et al. 2009. Distribution, prevalence, molecular typing, and virulence of Vibrio parahaemolyticus isolated from different sources in coastal province Jiangsu, China. Food Control 20: 907-912.

15. Maluping RP, Ravelo C, Lavilla-Pitogo CR, Krovacek K, Romalde JL. 2005. Molecular typing of Vibrio parahaemolyticus strains isolated from the Philippines by PCR-based methods. J. Appl. Microbiol. 99: 383-391.

16. Zhao F, Zhou DQ, Cao HH, Ma LP, Jiang YH. 2011. Distribution, serological and molecular characterization of Vibrio parahaemolyticus from shellfish in the eastern coast of China. Food Control 22: 1095-1100.

17. Marhual NP, Das BK, Samal SK. 2012. Characterization of Vibrio alginolyticus and Vibrio parahaemolyticus isolated from Penaeus monodon: antimicrobial resistance, plasmid profiles and random amplification of polymorphic DNA analysis. Afr. J. Microbiol. Res. 6: $4261-4269$

18. Li RC, Chiou JC, Chan EW, Chen S. 2016. A novel PCR-based approach for accurate identification of Vibrio parahaemolyticus. Front. Microbiol. 7: 44 .

19. Alaboudi AR, Ababneh M, Osaili TM, Shloul KA. 2016. Detection, identification, and prevalence of pathogenic Vibrio parahaemolyticus in fish and coastal environment in Jordan. J. Food Sci. 81: M130-M134.

20. Silva IP, Carneiro CS, Saraiva MAF, Oliveira TAS, Sousa OV, Evangelista-Barreto NS. 2018. Antimicrobial resistance and potential virulence of Vibrio parahaemolyticus isolated from water and bivalve mollusks from Bahia, Brazil. Mar. Pollut. Bull. 131: 757-762.

21. Xu XK, Wu QP, Zhang JM, Cheng JH, Zhang SH, Wu K. 2014. Prevalence, pathogenicity, and serotypes of Vibrio parahaemolyticus in shrimp from Chinese retail markets. Food Control 46: 81-85.

22. Caburlotto G, Gennari M, Ghidini V, Tafi M, Lleo MM. 2010. Serological and molecular characterization of Vibrio parahaemolyticus marine strains carrying pandemic genetic markers. ISME J. 4: 1071-1074.

23. Masatoshi O, Ro O, Akihiko T, Masatomo M, Eiji A, Haruo W. 2010. Genetic analyses of the putative $\mathrm{O}$ and $\mathrm{K}$ antigen gene clusters of pandemic Vibrio parahaemolyticus. Microbiol. Immunol. 52: 251-264.

24. Zhang YY, Mei LL, Zhang JY, Zhan L, Chen JC, Chen HH, et al. 2016. Triplex PCR method for Vibrio parahemolyticus serotype O3:K6 strains detection. Chinese I. Zoonoses 32: 632-635.

25. Yukiko HK, Kanji S, Mitsuaki N, Ashrafuzzaman C, Jun Y, Yoshimitsu O, et al. 2003. Prevalence of pandemic thermostable direct hemolysin-producing Vibrio parahaemolyticus O3:K6 in seafood and the coastal environment in Japan. Appl. Environ. Microbiol. 69: 3883-3891.

26. Ghenem L, Elhadi N. 2018. Isolation, molecular characterization, and antibiotic resistance patterns of Vibrio parahaemolyticus isolated from coastal water in the Eastern Province of Saudi Arabia. J. Water Health 16: 57-69.

27. Lou K, Ban R, Zhao XM. 2002. Curing of the Bacillus subtilis plasmid using sodium dodecyl sulfate. transactions of Tianjin University. 8: $148-151$.

28. Devi R, Surendran PK, Chakraborty K. 2009. Antibiotic resistance and plasmid profiling of Vibrio parahaemolyticus isolated from shrimp farms along the southwest coast of India. World J. Microbiol. Biotechnol. 25: 2005-2012.

29. Jiang YH, Chu YB, Xie GS, Li FL, Wang LZ, Huang J, et al. 2019. Antimicrobial resistance, virulence and genetic relationship of Vibrio parahaemolyticus in seafood from coasts of Bohai Sea and Yellow Sea, China. Int. J. Food Microbiol. 290: 116-124.

30. Pan H, Paudyal N, Li XL, Fang WH, Yue M. 2018. Multiple food-animal-borne route in transmission of antibiotic-resistant Salmonella Newport to humans. Front. Microbiol. 9: 23 .

31. Chen J, Zhang RH, Qi XJ, Zhou B, Wang JK, Chen Y, et al. 2017. Epidemiology of foodborne disease outbreaks caused by Vibrio parahaemolyticus during 2010-2014 in Zhejiang Province, China. Food Control 77: 110-115.

32. Sperling L, Alter T, Huehn S. 2015. Prevalence and antimicrobial resistance of Vibrio spp. in retail and farm shrimps in Ecuador. J. Food Prot. 78: 2089-2092.

33. Chen SY, Ge BL. 2010. Development of a toxR-based loop-mediated isothermal amplification assay for detecting Vibrio parahaemolyticus. BMC Microbiol. 10: 41

34. Chiou JC, Li RC, Chen S. 2015. CARB-17 family of $\beta$-lactamases mediates intrinsic resistance to penicillins in Vibrio parahaemolyticus. Antimicrob. Agents Chmother. 59: 3593-3595.

35. Han DS, Yu F, Tang H, Ren CL, Wu CY, Zhang P, et al. 2017. Spreading of pandemic Vibrio parahaemolyticus O3:K6 and its serovariants: a re-analysis of strains isolated from multiple studies. Front. Cell. Infect. Mi. 7: 188.

36. Hernándezdíaz LDJ, Leonsicairos N, Velazquezroman J, Floresvillaseñor H, Guadronllanos AM, Martinezgarcia JJ, et al. 2015. A pandemic Vibrio parahaemolyticus O3:K6 clone causing most associated diarrhea cases in the Pacific Northwest coast of Mexico. Front. Microbiol. 6: 221.

37. Ma C, Deng XL, Ke CW, He DM, Liang ZM, Li W, et al. 2014. Epidemiology and etiology characteristics of foodborne outbreaks caused by Vibrio parahaemolyticus during 2008-2010 in Guangdong Province, China. Foodborne Pathog. Dis. 11:21-29.

38. Zhang HZ, Sun SF, Shi WM, Cui L, Gu QF. 2013. Serotype, virulence, and genetic traits of foodborne and clinical Vibrio parahaemolyticus isolates in Shanghai, China. Foodborne Pathog. Dis. 10: 796-804.

39. Yang ZQ, Jiao XA, Zhou XH, Cao GX, Fang WM, Gu RX. 2008. Isolation and molecular characterization of Vibrio parahaemolyticus from fresh, low-temperature preserved, dried, and salted seafood products in two coastal areas of eastern China. Int. J. Food Microbiol. 125: 279-285.

40. Parvathi A, Kumar HS, Bhanumathi A, Ishibashi M, Nishibuchi M, Karunasagar I, et al. 2010. Molecular characterization of thermostable direct haemolysin-related haemolysin (TRH)-positive Vibrio parahaemolyticus from oysters in Mangalore, India. Environ. Microbiol. 8: 997-1004. 
41. Sahilah AM, Laila RAS, Sallehuddin HM, Osman H, Aminah A, Azuhairi AA. 2014. Antibiotic resistance and molecular typing among cockle (Anadara granosa) strains of Vibrio parahaemolyticus by polymerase chain reaction (PCR)-based analysis. World J. Microbiol. Biotechnol. 30: 649-659.

42. Tunung R, Jeyaletchumi P, Noorlis A, Tang YH, Sandra A, Ghazali FM, et al. 2012. Biosafety of Vibrio parahaemolyticus from vegetables based on antimicrobial sensitivity and RAPD profiling. Int. Food Res. J. 19: 467-474.

43. Vázquez-Sánchez D, López-Cabo M, Saá-Ibusquiza P, Rodríguez-Herrera JJ. 2012. Incidence and characterization of Staphylococcus aureus in fishery products marketed in Galicia (Northwest Spain). Int. J. Food Microbiol. 157: 286-296.

44. Noorlis A, Ghazali FM, Cheah YK, Zainazor TCT, Ponniah J, Tunung R, et al. 2011. Prevalence and quantification of Vibrio species and Vibrio parahaemolyticus in freshwater fish at hypermarket level. Int. Food Res. J. 18: 689-695.

45. Zarei M, Borujeni MP, Jamnejad A, Khezrzadeh M. 2012. Seasonal prevalence of Vibrio species in retail shrimps with an emphasis on Vibrio parahaemolyticus. Food Control 25: 107-109.

46. Kang CH, Shin YJ, Jang SC, Yu HS, Kim SK, An S, et al. 2017. Characterization of Vibrio parahaemolyticus isolated from oysters in Korea: Resistance to various antibiotics and prevalence of virulence genes. Mar. Pollut. Bull. 118: 261-266.

47. Letchumanan V, Yin WF, Lee LH, Chan KG. 2015. Prevalence and antimicrobial susceptibility of Vibrio parahaemolyticus isolated from retail shrimps in Malaysia. Front. Microbiol. 6: 33

48. Al-Hasnawy HH, Jodi MR, Hamza HJ. 2018. Molecular characterization and sequence analysis of plasmid-mediated quinolone resistance genes in extended-spectrum beta-lactamases producing uropathogenic Escherichia coli in Babylon Province, Iraq. Rev. Med. Microbiol. 29: 129-135.

49. Jiang Y, Yao L, Li F, Tan Z, Zhai Y, Wang L. 2014. Characterization of antimicrobial resistance of Vibrio parahaemolyticus from cultured sea cucumbers (A postichopus japonicas). Lett. Appl. Microbiol. 59: 147-154.

50. Xu XK, Cheng JH, Wu QP, Zhang JM, Xie TF. 2016. Prevalence, characterization, and antibiotic susceptibility of Vibrio parahaemolyticus isolated from retail aquatic products in North China. BMC Microbiol. 16: 32.

51. Zhao S, Ma LC, Wang Y, Fu GH, Zhou JF, Li XC, et al. 2018. Antimicrobial resistance and pulsed-field gel electrophoresis typing of Vibrio parahaemolyticus isolated from shrimp mariculture environment along the east coast of China. Mar. Pollut. Bull. 136: 164170.

52. Tunung R, Margaret S, Jeyaletchumi P, Chai LC, Tuan Zainazor TC, Ghazali FM, et al. 2010. Prevalence and quantification of Vibrio parahaemolyticus in raw salad vegetables at retail level. J. Microbiol. Biotechnol. 20: 391-396.

53. Daramola BA, Williams R, Dixon RA. 2009. In vitro antibiotic susceptibility of Vibrio parahaemolyticus from environmental sources in northern England. Int. J. Antimicrob. Agents 34: 499-500.

54. Shaw KS, Goldstein RER, He X, Jacobs JM, Crump BC, Sapkota AR. 2014. Antimicrobial susceptibility of Vibrio vulnificus and Vibrio parahaemolyticus recovered from recreational and commercial areas of Chesapeake bay and Maryland coastal bays. PLoS One 9 : e89616.

55. Yano Y, Hamano K, Satomi M, Tsutsui I, Ban M, Aue-Umneoy D. 2014. Prevalence and antimicrobial susceptibility of Vibrio species related to food safety isolated from shrimp cultured at inland ponds in Thailand. Food Control 38: 30-36.

56. Silvester R, Alexander D. 2015. Prevalence, antibiotic resistance, virulence and plasmid profiles of Vibrio parahaemolyticus from a tropical estuary and adjoining traditional prawn farm along the southwest coast of India. Ann. Microbiol. 65: 2141-2149.

57. Zhang RF, Wang YL, Gu JD. 2006. Identification of environmental plasmid-bearing Vibrio species isolated from polluted and pristine marine reserves of Hong Kong, and resistance to antibiotics and mercury. Antonie Van Leeuwenhoek 89: 307-315. 